\title{
Evolution \& Explosion of Massive Stars Leading to IIP-IIL SNe with MESA \& SNEC
}

\author{
Sanskriti Das ${ }^{1}$ and Alak Ray ${ }^{2}$ \\ ${ }^{1}$ Department of Physics, Indian Institute of Technology Bombay, Mumbai- 400076, India \\ email: dassanskriti@gmail.com \\ ${ }^{2}$ Dept. of Astronomy \& AstroPhysics, Tata Institute of Fundamental Research, Mumbai- \\ 400005,India \\ email: akr@tifr.res.in
}

\begin{abstract}
We show how the dense shells of circumstellar gas immediately outside the red supergiants(RSGs) can affect the early optical light curves of Type II-P SNe taking the example of 2013ej. The peak in V, R and I bands, decline rate after peak and plateau length are found to be strongly influenced by the dense CSM formed due to enhanced mass loss during the oxygen and silicon burning stage of the progenitor. We find that the required explosion energy for the progenitors with CSM is reduced by almost a factor of 2 .
\end{abstract}

Keywords. Supernovae, hydrodynamics, radiative transfer, II-P, II-L, CSM, individual: 2013ej

\section{Introduction}

Massive stars (ZAMS mass $>8 M_{\odot}$ ) end up their lives through core-collapse supernovae (CCSNe). Type-II SNe, a class of CCSNe are identified by the P-cygni profile of hydrogen in their early spectra. Type II-P SNe cover a large fraction $(\sim 48 \%)$ of the whole type-II SNe population (Smith et al. 2011). These have pronounced plateaus in their visible band light curves that remain within 1 mag of maximum brightness for an extended period e.g. 60-100 rest frame days and is followed by exponential tail at late times. II-Ls were originally separately classified by their brighter peak and linearly falling luminosity after peak, but Sanders et al. 2015 shows that II-P and II-L form a continuous and statistically indistinguishable class of CCSNe. There has been search for a range of progenitors producing a continuous transition from II-P to II-L by arguing the qualitative similarity in their light curve pattern but so far it has not been possible to simulate II-L as an extreme case of II-P SNe (Morozova et al. 2015). Type II-n SNe show narrow emission lines of $\mathrm{H}-\alpha$ due to the interaction of shock and the stellar ejecta with the circumstellar medium. So far, II-P/II-L and II-n have been kept into separate categories by virtue of their observed characteristics and inferred progenitor properties. Recently there has been considerations on the effect on the early light curves of II-P/II-L SNe due to dense circumstellar material immediately outside the progenitors (Valenti et al. 2015; Morozova et al. 2017; Yaron et al. 2017) and a subclass of moderately interacting SNe has been identified. This may act as an intermediate of II-P/II-L and II-n and fill the gap between observed ZAMS (Zero Age Main Sequence) mass range of their respective progenitors(Moriya et al. 2011).

We have evolved the stars in MESA (Modules for Experiments in Stellar Astrophysics)(Paxton et al. 2011,Paxton et al. 2013,Paxton et al. 2015) since their pre-ZAMS stage till the Fe core collapse with a history of enhanced mass loss rate in last few years, and constructed the CSM from that information. Our method is self-consistent in two 

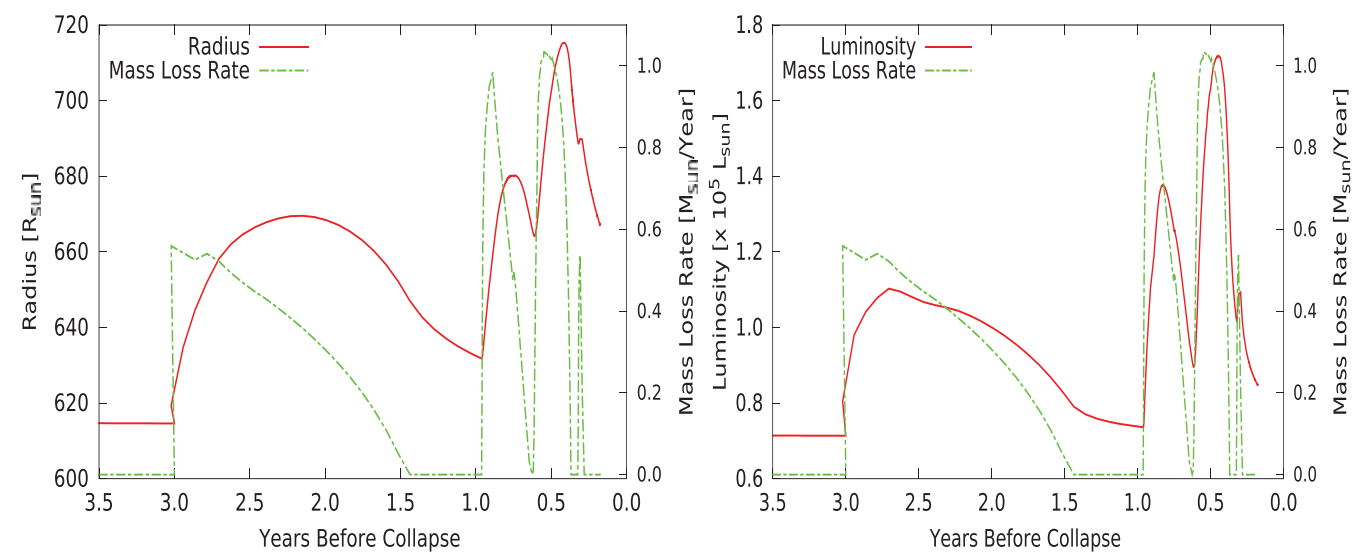

Figure 1. Variation in luminosity and radius with large mass loss rate in late stage of stellar evolution of a model of ZAMS mass $13 M_{\odot}$.

ways: firstly, since the simulation of enhanced mass loss over a timescale of few years reveals the impact on surface properties (mainly luminosity and radius) naturally from the evolution (Fig. 1), the pre-SN progenitor carries the trace of the event with it and secondly, the modelling of the CSM also accounts for these changes in the star, and thus the CSM profile becomes more realistic. This is in contrast with the earlier works on the effect of CSM on II-P SNe where artificially designed CSM profile is stitched to the pre-SN star independently modelled from stellar evolution codes (e.g. KEPLER) without any huge mass loss history (Morozova et al. 2017; Moriya et al. 2017). The progenitor with CSM is then exploded via SNEC (SuperNova Explosion Code)(Morozova, Ott \& Piro 2015) and the light curve is compared against the light curve of the progenitor with no excess mass loss history and hence no dense CSM in the immediate vicinity of the exploding star. We make a comparative study of the models best fitting the optical light curve of Type II-P SN2013ej (sometimes described as II-L because of fast decline of 1.74 mag/100 days in intermediate stage) and show in next section how the models with CSM improves the fitting.

\section{Circumstellar medium structure and explosion characteristics}

To study the effect of CSM in the context of SN2013ej we have taken $0.295 Z_{\odot}$ metallicity, which is the equal to the metallicity of the nearby HII region number 197 of Cedrés et al. 2012 and ZAMS mass range of 12-19 $M_{\odot}$ for all of our MESA models. We use Ledoux criterion for convection, and take mixing length parameter $\alpha_{m l t}=2$, exponentially decreasing overshooting (both for the core and the convective shells) with $f_{o v}=$ 0.025 and $f_{0}=0.05$, and semi-convection efficiency $\alpha_{s c}=0.1$. Near the center of the star we increase mass resolution with mesh_delta_coeff_for_highT $=1$. During continuous mass ejection temporal resolution has been kept to default value with varcontrol_target $=10^{-4}$. The resolution is increased by using max_timestep $=0.05 \times 10^{7}$ seconds during the episodes of enhanced mass loss rate.The average mass loss rate has been kept to $\sim 10^{-6} M_{\odot} y r^{-1}$ (Vink's scheme for hot $\left(T_{s}>10^{4} \mathrm{~K}\right.$ ) with wind $\eta=1$ and Dutch scheme for cool wind with $\eta=1$ and 0.5 ) constrained by X-ray studies which has probed mass loss rate in the range of 40-400 years before the explosion (Chakraborti et al. 2016). The enhanced mass loss rate was triggered by hydrogen stripping (remove_H_wind_mdot) and flashes (flash_wind_mdot) with an upper limit of $\sim 1 M_{\odot} y r^{-1}$ in last $\sim 3$ years of 

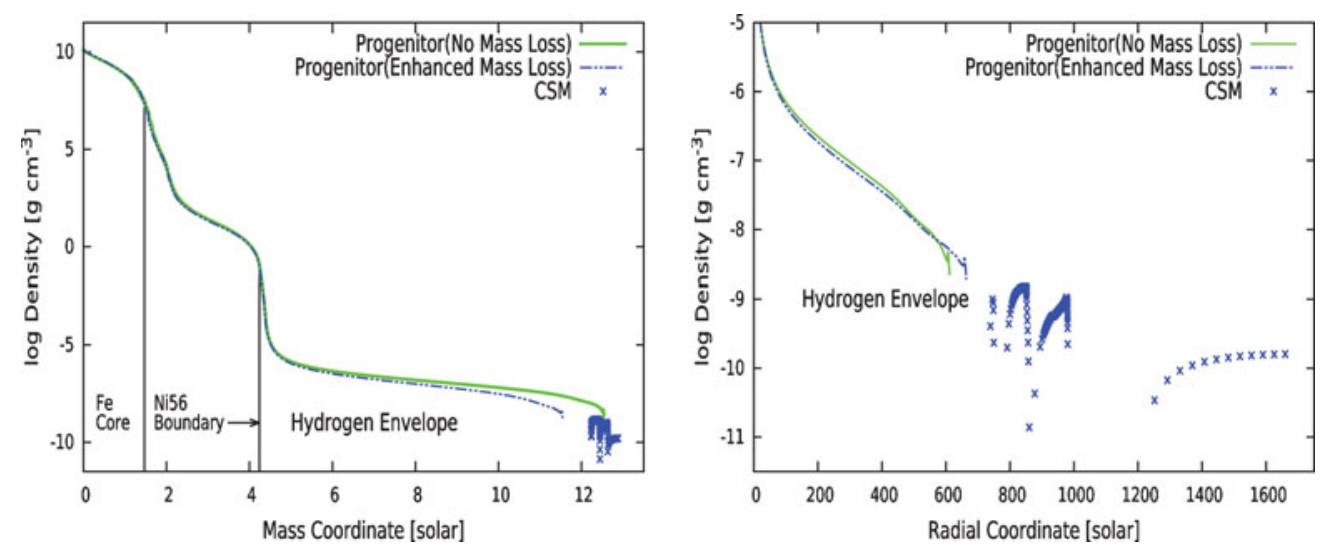

Figure 2. Pre-SN density profile of a progenitor of ZAMS mass $13 M_{\odot}$. The model without enhanced mass loss and the one with shells of CSM have been shown in same plot for comparison. The radial profile of the central part of $\sim 4.5 M_{\odot}$ has been excluded from the plot on right side to show the CSM with better resolution.

evolution. Any heavy mass loss much before this would move away too far and become dilute to substantially affect the early light curves. Noting that the bolometric luminosity and radius vary with mass loss rate with certain correlation (Fig. 1), the CSM profile has been calculated from the history of mass loss rate, radius and surface temperature assuming asymptotic wind speed of $10 \mathrm{~km} / \mathrm{s}$. Although the launch velocity was found to be correlated with mass loss rate, we assume the ejected gas has had enough time to relax to the asymptotic speed during the episodic ejection. The composition in CSM is assumed to be the same as that of the surface. In contrast to a smooth continuous inverse square radial profile of density our constructed CSM is a collection of discrete shells of non-monotonically varying density. The resultant CSM has been stitched to the progenitor (e.g. Fig. 2) and exploded via SNEC using a thermal bomb. The explosion energy has been varied in the range of $0.4-1.4 \times 10^{51} \mathrm{erg} .{ }^{56} \mathrm{Ni}$ mass has been kept fixed at $0.0207 M_{\odot}$ and evenly spread from outside the Fe core to the middle of He core (Fig. 2). After boxcar smoothing this distribution gets modified with a tail extending upto the He core boundary. The mass of the NS has been taken to be equal to Fe core mass. The light curves have been calculated till 120 days to cover a part of the radioactive tail after plateau. The magnitude in different bands have been calculated using proper bolometric corrections with blackbody approximation.

The resultant light curves in $\mathrm{V}, \mathrm{R}$ and $\mathrm{I}$ bands have been compared with the data given by Richmond 2014 and Yuan et al. 2016. $\dagger$ The reduced error of our SNEC outputs against the data has been defined following Morozova et al. 2017 as

$$
\chi_{\nu}^{2}=\sum_{V, R, I} \sum_{t_{i} \leqslant t_{p l}} \frac{\left(m_{\text {obs }}\left(t_{i}\right)-m_{t h}\left(t_{i}\right)\right)^{2}}{\sigma_{i}^{2}} \times \frac{1}{\left(n_{V}+n_{R}+n_{I}-3\right)}
$$

Here $t_{p l}$ is plateau length which is $\sim 99$ days $\ddagger, m_{t h}=M_{t h}+$ (distance and extinction correction), $n_{\lambda}$ is no. of data points in a particular band. All sources of errors i.e. the photometric error in observational data, uncertainty in distance and extinction estimation

$\dagger$ Since SNEC does not calculate Fe line blanketing in U and B bands, these bands are excluded from goodness of fit analysis.

$\ddagger$ By fitting the V-band light curve with the function $y(t)=-a_{0} /\left(1+\exp \frac{t-t_{p l}}{w_{0}}\right)+\left(p_{0} \times t\right)+m_{0}$ as proposed in Valenti et al. 2016, the best fit value of the plateau duration is $t_{p l}=98.77$ days 

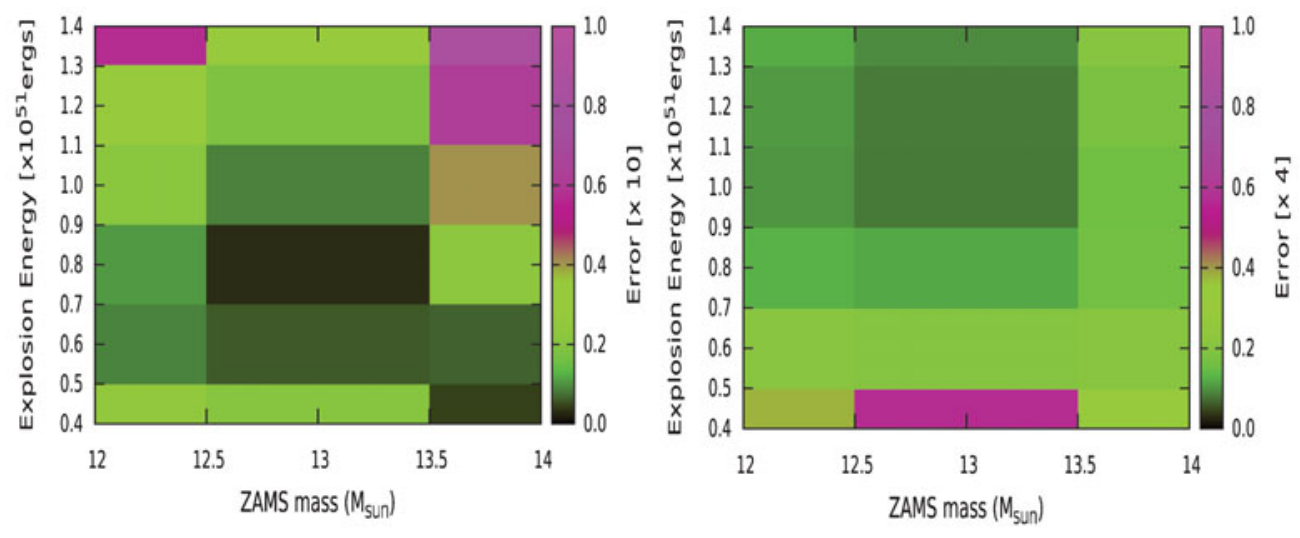

Figure 3. Error maps of the models of different ZAMS mass and explosion energy against V,R and I bands of SN2013ej (data: Yuan et al. 2016). The photometric error in observational data, errors in estimation of the distance of host galaxy and average galactic extinction (taken from Huang et al. 2015) have been included in the error analysis.

have been included in $\sigma$. We have used the distance and interstellar extinction correction quoted in Richmond 2014, Bose et al. 2015, Huang et al. 2015 and Yuan et al. 2016. We find that for the set of values quoted in Richmond the best fitted model with CSM has ZAMS mass $12-13 M_{\odot}$ and explosion energy $E_{\text {exp }}=0.6 \times 10^{51} \mathrm{ergs}$, while the values of Yuan and Bose predict best fitted ZAMS mass $=13 M_{\odot}$ and a range of $E_{\exp }=$ $0.6-0.8 \times 10^{51} \mathrm{ergs}$. Huang has considered a larger value of extinction by taking the reddening of host galaxy M74 into account. Using their values we can pinpoint a ZAMS mass $13 M_{\odot}$ and $E_{\text {exp }}=0.8 \times 10^{51} \mathrm{ergs}$. The explosion of models without CSM deviate from the data with a different distribution (Fig. 3), and with an error larger by a factor of 6-8 than explosions powered by the circumstellar medium (Table 1). The results have been cross-checked against the data from Valenti et al. 2014 and Bose et al. 2015 as well. It turns out that the model with CSM best fitted using Huang's values is visually closest to the data (Fig. 4). It strengthens the consideration of host extinction which is confirmed by recent analysis of massive star population around the site of explosion(Maund 2017). Although it was intended to fit upto the plateau only, since SNEC is not reliable beyond that region (Morozova, Ott \& Piro 2015), the model with dense and nearby CSM fits the radioactive tail reasonably well in three bands. Also, the model predicts the peak magnitude in $\mathrm{U}$ and $\mathrm{B}$ bands correctly. On the other hand, the best-fit model without CSM hardly satisfies any feature of the light curve. This analysis is consistent with the spectroscopic observations of Bose et al. 2015 where a weak ejecta-CSM interaction was inferred from the high velocity $H \alpha-H \beta$ profiles. This suggests that the progenitor of SN2013ej was surrounded by dense CSM and supports the suggestion of Smith \& Arnett 2014 of heavy mass loss immediately prior to the SN explosion.

\section{Discussion}

In the very late stage of post-main-sequence evolution of RSGs the mass loss rate can increase by several orders of magnitude (Quataert \& Shiode 2012; Smith \& Arnett 2014; Moriya 2014). This can give rise to a dense and slowly moving circumstellar gas just outside the star. Once the shock comes out of the star it hits onto the CSM and deposits a larger fraction of its energy to the CSM which gets diffusively radiated and brightens the early optical light curves by degrading the adiabatic loss. 
Table 1. Overview of progenitor and explosion properties best-fitted for SN2013ej

\begin{tabular}{ccccccc}
\hline $\begin{array}{c}\text { ZAMS } \\
\text { mass }\left[M_{\odot}\right]\end{array}$ & $\begin{array}{c}\text { Pre-SN } \\
\text { mass }\left[M_{\odot}\right]\end{array}$ & $\begin{array}{c}\text { CSM } \\
\text { mass }\left[M_{\odot}\right]^{1}\end{array}$ & $\begin{array}{c}\text { Pre-SN } \\
\text { radius }\left[R_{\odot}\right]\end{array}$ & $\begin{array}{c}\text { CSM } \\
\text { radius }\left[R_{\odot}\right]^{2}\end{array}$ & $\begin{array}{c}\text { Energy } \\
{\left[10^{51} \text { ergs }\right]^{3}}\end{array}$ & $\begin{array}{c}\text { Goodness of Fit } \\
{\left[\chi_{\nu}^{2}\right]}\end{array}$ \\
\hline 13 & 11.60 & 0.76 & 667 & 1650 & $0.6-0.8$ & $0.0019-0.0028$ \\
13 & 12.36 & - & 617 & - & $1.0-1.2$ & $0.0124-0.0227$ \\
\hline
\end{tabular}

\section{Notes:}

${ }^{1}$ lost mass during enhanced mass loss rate in last $(\sim 2-3)$ few years.

2 the external radius of the dense CSM formed in the last few years before explosion

3 asymptotic energy of the shock after breakout

$4{ }^{56} \mathrm{Ni}$ mass was kept fixed at $0.021 M_{\odot}$

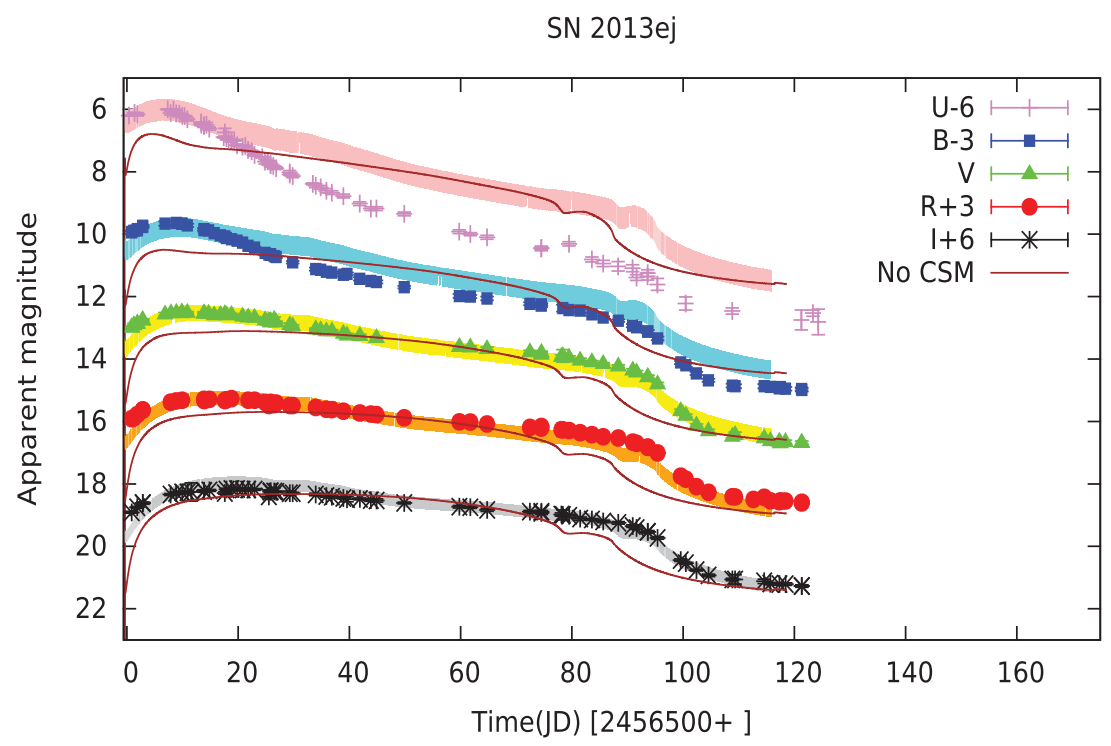

Figure 4. The UBVRI light curves of SN2013ej (data: Yuan et al. 2016). The curves for best-fitted model (fitted against VRI bands till the plateau of $\sim 99$ days) with CSM are shown with the errors at each point. Values of distance and extinction estimation have been taken from Huang et al. $2015\left[d=9.6 \pm 0.5 \mathrm{Mpc}, A_{V}=0.37 \pm 0.19\right.$ with relative extinction relation borrowed from Cardelli et al. 1989]. The best-fitted model without CSM is shown in thin lines for the sake of comparison.

Here we have discussed how the same star can pass through two evolutionary tracks differing in a timescale much less than the Kelvin-Helmholtz timescale and end up as different pre-SN progenitors, although occupying roughly the same position in H-R diagram. The density, velocity and temperature profiles of hydrogen envelope of the stars with no huge mass loss history are substantially different from those of the progenitors surrounded by dense and compact CSM (Fig.2); even though total mass (envelope + CSM) is same in both cases. So the rise and plateau of the light curve, which are functions of hydrogen profile are expected to be different. In these cases, although the pre-SN images cannot be distinguished, the light curves of explosion will differ. We note some particular differences in the presence of dense CSM: UV and optical bands are simultaneously bright in early phase, for a fixed shock energy the optical light curve is brighter at peak, flatter after peak and the plateau is longer. The dense and compact CSM behaves 
like an unbound extended part of the progenitor itself. The key difference between this scenario and usual II-n SNe is that the extended CSM in II-n is optically thin, while here the photosphere lies in the compact CSM for $\sim 40$ days since explosion. In usual II-n the interactive phase lasts for $\sim 1$ yr but in our case that is $\sim 2$-3 days and the breakout happens when the shock has already traversed through the CSM. Radio and flash-ionization non-detection early on can constrain the radial extent of the CSM and confirm proximity and compactness (Yaron et al. 2017).

\section{References}

Bose, S. et al. 2015, ApJ, 806, 160, 18 pp.

Cardelli, J. A., Geoferey, G. C. \& Mathis, J. S., 1989, ApJ, 345:245-256

Cedrés, B., Cepa, J., Bongiovanni, Á. et al. 2012, A\& A, 545, A43

Chakraborti, S. et al. 2016, ApJ, 817, 22, 8 pp.

Fraser, M. et al. 2014, MNRAS, 439, L56-L60

Huang, F. et al. 2015, ApJ, 807, 59, 12 pp.

Maund, J. R., 2017, MNRAS, 2017arXiv170401957M

Moriya, T., Tominaga, N., Blinnikov, S. I., Baklanov, P. V., \& Sorokina, E. I., 2011, MNRAS, $415,199-213$

Moriya, T., 2014, A\& A, 564, A83

Moriya, T. J., Yoon, S. C., Gräfener, G., \& Blinnikov, S. I., 2017arXiv170303084M

Morozova, V., Ott, C. D., \& Piro, A. L., 2015, Astrophysics Source Code Library, record ascl:1505.033

Morozova, V. et al. 2015, ApJ, 814, 63, 18 pp.

Morozova, V., Piro, A. L. \& Valenti, S. 2017, ApJ, 838, 28, 12 pp.

Paxton, B. et al. , 2011, ApJS, 192, 3, 35 pp.

Paxton, B. et al. , 2013, ApJS, 208, 4, 42 pp.

Paxton, B. et al. , 2015, ApJSS, 220, 15, 44 pp.

Quataert, E. \& Shiode, J., 2012, MNRAS, 423, L92 - L96

Richmond, M. W., 2014, JAAVSO, 42, 333

Sanders, N. E. et al. 2015, ApJ, 799, 208, 23 pp.

Smith, N. et al. 2011, MNRAS, 412, 1512

Smith, N. \& Arnett, D. W., 2014, ApJ, 785, 82, 12 pp.

Valenti, S. et al. 2014, MNRAS, 438, L101-L105

Valenti, S. et al. 2015, MNRAS, 448, 2608-2616

Valenti S. et al. 2016, MNRAS, 459, 3939-3962

Yaron, O. et al. 2017, Nature Physics, 4025

Yuan, F. et al. 2016, MNRAS 461, 2003 - 2018 